\title{
String propagation in non-compact backgrounds
}

\author{
Carmen A. Núñez ${ }^{* \dagger}$ \\ Instituto de Astronomía y Física del Espacio (IAFE) \\ C.C. 67 - Suc. 28, 1428 Buenos Aires, Argentina \\ and \\ Physics Department, University of Buenos Aires \\ E-mail: carmen@iafe.uba.ar
}

\begin{abstract}
In this lecture we review some recent developments in non-rational conformal field theory, which allow a description of strings moving in non-compact backgrounds. In particular, we discuss recent progress in the $S L(2, \mathbb{R})$ WZW model, which describes string propagation in $A d S_{3}$.
\end{abstract}

Fifth International Conference on Mathematical Methods in Physics - IC2006

April 24 - 282006

Centro Brasileiro de Pesquisas Fisicas (CBPF/MCT), Rio de Janeiro, Brazil

\footnotetext{
*Speaker.

$\dagger$ It is a pleasure to thank the organizers for a very stimulating conference. I would also like to express my gratitude to my collaborators E. Herscovich, S. Iguri, P. Minces and L. Nicolás, with whom most of the material contained in this review has been discussed and ellaborated.
} 


\section{Introduction}

Several aspects of spacetime physics in string theory have been made accessible due to the development of world-sheet methods. In particular, the significant progress of algebraic techniques in rational conformal field theory (RCFT) has been crucial in the advancement of knowledge in string compactification and string phenomenology. Viceversa, motivated by the need to understand nontrivial string backgrounds, many powerful results have been obtained in RCFT, with applications in other areas of physics as well, mainly to statistical systems at criticality.

Strings propagating in compact target spaces have discrete spectra. This feature simplifies the analysis of the underlying world-sheet models and allows to solve the theory using algebraic tools. More recent developments of string theory, such as the AdS/CFT correspondence, little string theory, cosmological and time dependent processes, however, require to consider non-compact backgrounds which lead to continuous spectra. In this case, the algebraic methods of RCFT have to be replaced by more intricate analytic techniques. In this lecture I will discuss some recent advancements beyond the rational cases which allow a description of closed strings moving in noncompact target spaces. Unlike compact backgrounds, where many model independent results have been obtained, the studies of non-compact target spaces are restricted so far mainly to two models: Liouville theory and the $S L(2, \mathbb{R})$ WZW model. They both have very important applications to gravity and black hole physics in two and three dimensions. The first one describes strings moving in an exponential potential with a non-constant dilaton and the second one represents strings in three dimensional $A d S$ spacetime $\left(A d S_{3}\right)$. These examples are the non-rational analogues of the minimal and $S U(2)$ WZW models, which have been crucial for the development of RCFT and its applications to string theory.

The first part of this short review is based on the complete and comprehensive lecture notes by V. Schomerus [1] on general aspects of non-rational CFT and non-compact string backgrounds, and the second part summarizes results obtained in references [2] - [6] for the $S L(2, \mathbb{R})$ WZW model.

\section{String propagation in background fields}

The world-sheet of strings propagating in any target space with background metric $G$ is described by the following non-linear sigma model action

$$
S[X]=\frac{1}{4 \pi \alpha^{\prime}} \int_{\Sigma} d^{2} z G_{\mu \nu} \partial X^{\mu} \bar{\partial} X^{v}+\cdots
$$

where $X^{\mu}(z, \bar{z})$ are $D$-dimensional spacetime coordinates, $\mu, v=0, \cdots, D-1, \Sigma$ denotes the compact topology of the world-sheet and the dots stand for other non-trivial background fields that can appear, such as dilaton, antisymmetric tensor or gauge fields.

String interactions are described by correlation functions in the theory (1) and they may be computed, at least in principle, using Feynman path integrals. The remarkable success of two dimensional RCFT, however, was mainly based on a different approach that systematically exploits the representation theory of certain infinite dimensional symmetries, known as chiral algebras. Strings moving in flat space already exhibit such symmetry algebra. In this case, the equations 
of motion for the fields $X^{\mu}$ are the wave equations $\partial \bar{\partial} X^{\mu}=0$, which imply the existence of a holomorphic current $J^{\mu}(z)=\partial X^{\mu}=\sum_{n} \alpha_{n}^{\mu} z^{-n-1}-$ and similarly an antiholomorphic $\bar{J}^{\mu}(\bar{z})$. The canonical commutation relations for the bosonic fields $X^{\mu}$, determine the simplest example of infinite dimensional chiral algebra, namely

$$
\left[\alpha_{m}^{\mu}, \alpha_{n}^{v}\right]=m \delta_{m+n} \eta^{\mu v}
$$

When non-trivial background fields are turned on, more general algebras appear, and the string scattering amplitudes are built from their representation theoretic data along with structure constants of the various operator product expansions.

Constructing the representation theoretic data is essentially a mathematical problem, which is the same for all models that possess the same chiral symmetry. The most important notions in the representation theory of chiral algebras include the set of representations, modular transformations, fusion of representations and the fusing matrix. The fields in the theory can be considered as operators on the state space of the model $\mathscr{H}$, which admits a decomposition into irreducible representations of the two commuting chiral algebras, $\mathscr{H}=\oplus_{i, \bar{i}} \mathscr{Y}_{i} \otimes \overline{\mathscr{V}}_{\bar{i}}$. For non-compact backgrounds the momenta are typically continuous and the sums have to be replaced by integrals. Particularly important are the fields associated with ground states of $\mathscr{H}$. We shall denote them by $\Phi_{i \bar{i}}$ and call them primary fields. All other fields in the theory can be obtained multiplying the primary fields with chiral fields and their derivatives.

The additional necessary data to characterize a string background are encoded in the short distance singularities of correlation functions

$$
\Phi_{i \bar{i}}\left(z_{1}, \bar{z}_{1}\right) \Phi_{j \bar{j}}\left(z_{2}, \bar{z}_{2}\right)=\sum_{n, \bar{n}} C_{i \bar{i}, j \bar{j}}^{n \bar{n}} z_{12}^{h_{n}-h_{i}-h_{j}} \bar{z}_{12}^{\bar{h}_{n}-\bar{h}_{i}-\bar{h}_{j}} \Phi_{n, \bar{n}}\left(z_{2}, \bar{z}_{2}\right)+\cdots
$$

where the numbers $C_{\bar{i}, \bar{j} j \bar{j}}^{n, \bar{n}}$ describe the scattering amplitude for three closed string modes. These triple couplings are determined from associativity of the OPE and crossing symmetry of the four point functions. They encode the full information about the closed string background since all higher scattering diagrams can be cut into such 3-point vertices.

Furthermore one can introduce the characters of the representations, and the full set of characters have the remarkable property to close under modular transformations.

RCFT have been systematically understood in the terms described above. Many classification problems have been solved (of which the classification of minimal models and $S U(2)$ modular invariant partition functions are simple examples) and the algebraic structure that underlies them has been uncovered: their highest weight representations, characters, fusion rules, structure constants, etc., are known. For non-unitary RCFT our understanding has advanced less, but partial results have been obtained. In particular, in all these theories a lot is known about the relation between the modular data and the fusion of representations as encoded in the OPE. An important relation is given by the Verlinde formula, which encodes the fact that the modular S-matrix diagonalizes the fusion matrix.

The presence of singular vectors in the Verma modules of the primary states is a relevant ingredient in the resolution of this program, since the requirement of null vector decoupling leads to differential equations for correlation functions, which allow to completely solve the theory in 
the simplest cases of the minimal [7] and the $S U(2)$ WZW models [8]. Alternatively, the Coulomb gas construction developed by Feigin and Fuchs [9] and by Dotsenko and Fateev [10] gives a more practical prescription to compute expectation values and, here again, the singular vectors provide the formal mathematical basis for the background charge formalism [11,12]. This method has successfully reproduced the fusion rules and expectation values of physical states in several rational theories. It has also allowed to explicitly construct and analyze various compact string backgrounds, such as the Gepner models.

Nowadays the possibility of extending the systematic understanding gained in RCFT to nonRCFT in order to describe non-compact string backgrounds is under active investigation. In the following sections I will discuss which of the algebraic structures discovered in RCFT can be extended to one particular non-rational example, the $S L(2, \mathbb{R})$ WZW model describing strings propagating in a three dimensional $A d S$ background. Ultimately one would certainly like to understand strings moving in $A d S_{5}$. But unfortunately, this goes far beyond the present technology, mainly because consistency of the $A d S_{5}$ background requires to turn on a RR 5-form field. The situation is somewhat better in $A d S_{3}$, where consistency may be achieved by switching on a NS-NS 3-form field strength $H$.

\section{3. $S L(2, R)$ WZW model and string theory on $A d S_{3}$}

A specially interesting application of the $S L(2, \mathbb{R})$ WZW model is the description of strings propagating in $\mathrm{AdS}_{3}$. The AdS/CFT correspondence provided new insights into this theory, initially considered in this context using worldsheet techniques in the free field approximation [13]. The construction of the exact theory was started in [14] and continued in a series of seminal papers in [15] - [17]. However, despite the important progress achieved in recent years, the theory has not been completely solved yet, mainly because $\mathrm{AdS}_{3}$ is non-compact and the worldsheet CFT is non-rational. In particular, unitarity has not been proven yet in all the sectors of the theory.

Let us start by reviewing the non-linear sigma model action which describes this theory. The metric of Euclidean $\mathrm{AdS}_{3}$, the hyperbolic space $\mathrm{H}_{3}^{+}$, can be written in Poincaré coordinates as

$$
d s^{2}=l^{2}\left(d \phi^{2}+e^{2 \phi} d \gamma d \bar{\gamma}\right)
$$

where $\phi \in \mathbb{R},\{\gamma, \bar{\gamma}\}$ are complex coordinates parametrizing the boundary of $H_{3}^{+}$, which is located at $\phi \rightarrow \infty$, and the parameter $l$ is related to the scalar curvature as $\mathscr{R}=-2 / l^{2}$. Consistent string propagation in this background metric requires in addition an antisymmetric rank two tensor background field $B=l^{2} e^{2 \phi} d \gamma \wedge d \bar{\gamma}$. The theory is described by the action

$$
S=\frac{k}{8 \pi} \int d^{2} z\left(\partial \phi \bar{\partial} \phi+e^{2 \phi} \bar{\partial} \gamma \partial \bar{\gamma}\right),
$$

where $k=l^{2} / l_{s}^{2}$ and $l_{s}$ is the fundamental string length. This non-linear sigma model is equivalent to a WZW model on $S L(2, \mathbb{R})$ (or actually its Euclidean version $S L(2, \mathbb{C}) / S U(2)$ ). The $S L(2, \mathbb{R})$-currents can be expanded in Laurent series $J^{a}(z)=\sum_{n=-\infty}^{\infty} J_{n}^{a} z^{-n-1}, a= \pm, 3$, and the coefficients satisfy the following chiral symmetry algebra

$$
\left[J_{n}^{a}, J_{m}^{b}\right]=i \varepsilon_{c}^{a b} J_{n+m}^{c}-\frac{k}{2} \eta^{a b} n \delta_{n+m, 0}
$$


where the Cartan Killing metric is $\eta^{+-}=\eta^{-+}=2, \eta^{33}=-1$. And similarly for the antiholomorphic currents. The Sugawara stress-energy tensor is given by

$$
T=\frac{\eta_{a b}}{k-2}: J^{a}(z) J^{b}(z):
$$

and it leads to a Virasoro algebra with central charge $c=\frac{3 k}{k-2}$.

The classical solutions of this theory were presented in [15]. Timelike geodesics oscilate around the center of $A d S_{3}$ whereas spacelike geodesics representing tachyons travel from one side of the boundary to the opposite. Solutions describing string propagation are obtained from the dynamics of pointlike particles through the spectral flow operation. Timelike geodesics give rise to short strings, bound states trapped in the gravitational potential of $A d S_{3}$. Conversely, long strings arising from spacelike geodesics can reach the boundary of $A d S_{3}$. The spectral flow parameter $w$ is an integer named winding number. Different values of $w$ correspond to distinct solutions, even at the classical level (as exhibited, for instance, by the energy spectrum).

At the quantum level, the building blocks of the Hilbert space $\mathscr{H}$ are unitary hermitic representations of $S L(2, \mathbb{R})$. The states $\mid j, m>$ satisfy

$$
C_{0}|j, m>=j(j-1)| j, m>\quad, \quad J_{0}^{3}|j, m>=m| j, m>, \quad J_{0}^{ \pm}|j, m\rangle=(m \mp j)|j, m \pm 1\rangle,
$$

with $\{m \in \mathbb{R}, j \in \mathbb{R}\} \vee\left\{m \in \mathbb{R}, j \in-\frac{1}{2}+i \mathbb{R}\right\}$ as required by hermiticity, and $J_{n}^{a}|j, m\rangle=0, n>0$.

The allowed representations are:

- Discrete lowest and highest weight representations

$$
\mathscr{D}_{j}^{ \pm}=\{|j, m\rangle ; j \in \mathbb{R} ; m= \pm j, \pm j \pm 1, \pm j \pm 2, \ldots\}
$$

- Principal continuous representation

$$
\mathscr{C}_{j}^{\alpha}=\left\{|j, m\rangle ; \quad j=\frac{1}{2}+i \lambda ; \lambda \in \mathbb{R} ; \quad m=\alpha, \alpha \pm 1, \alpha \pm 2, \ldots ; \alpha \in \mathbb{R}\right\}
$$

For applications to string theory one considers the universal cover of $\operatorname{SL}(2, \mathbb{R})$, where $j$ is not quantized. Notice that the vectors in $\mathscr{H}$ related by $j \leftrightarrow 1-j$ represent the same physical state and therefore $j$ can be restricted to $j \geq \frac{1}{2}$. The complete basis of $\mathscr{L}^{2}\left(A d S_{3}\right)$ is given by $\mathscr{C}_{j=-1 / 2+i \lambda}^{\alpha} \times \mathscr{C}_{j=-1 / 2+i \lambda}^{\alpha}$ and $\mathscr{D}_{j}^{ \pm} \times \mathscr{D}_{j}^{ \pm}$with $j>1 / 2$.

The representation space can be enlarged by acting on the primary states in these series with $J_{n}^{a}, n<0$. The corresponding representations are denoted by $\widehat{\mathscr{D}}_{j}^{ \pm}, \widehat{\mathscr{C}}_{j}^{\alpha}$. They are called positive energy representations since their $L_{0}$ eigenvalues are bounded below. The weight diagram of $\hat{\mathscr{D}}_{j}^{+}$ is shown in Figure 1.

Furthermore the full representation space contains the spectral flow images of these series, which correspond to winding classical strings. Actually the spectral flow operation leads to the following automorphism of the $S L(2, \mathbb{R})$ currents and Virasoro generators

$$
J_{n}^{3} \rightarrow \widetilde{J}_{n}^{3}=J_{n}^{3}-\frac{k}{2} w \delta_{n, 0} \quad, \quad J_{n}^{ \pm} \rightarrow \widetilde{J}_{n}^{ \pm}=J_{n \pm w}^{ \pm}, \quad L_{n} \rightarrow \widetilde{L}_{n}=L_{n}+w J_{n}^{3}-\frac{k}{4} w^{2} \delta_{n, 0}
$$

with $w \in \mathbb{Z}$. 


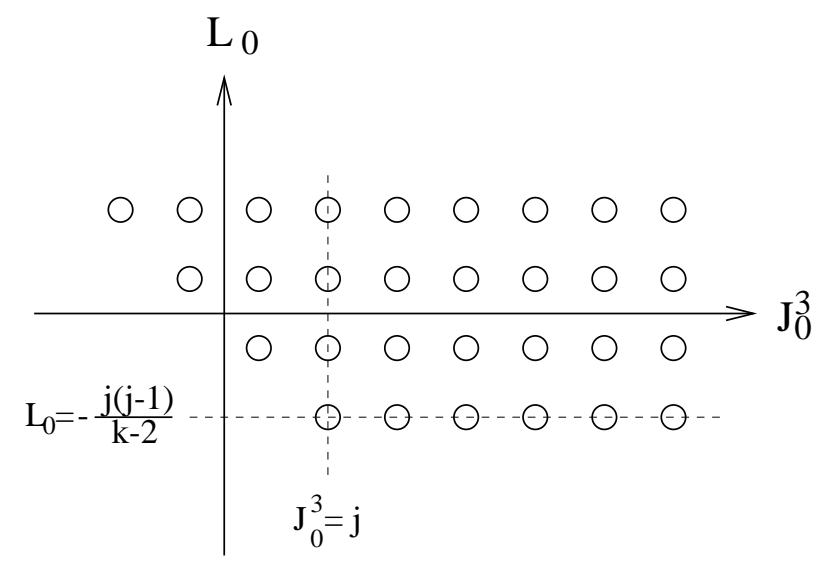

Figure 1: Weight diagram of the representation $\widehat{\mathscr{D}}_{j}^{+}$, whose the primary states form a discrete lowest weight representation $\mathscr{D}_{j}^{+}$.

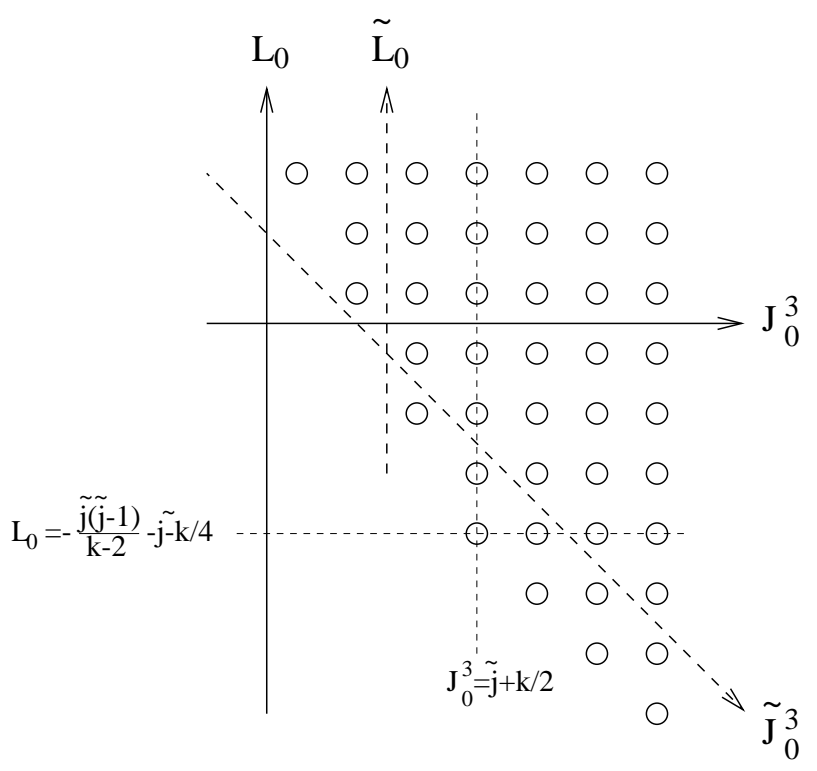

Figure 2: The spectral flow of Figure 1 with $w=1$

Unlike the compact $S U(2)$ case, the new operators generate inequivalent representations of $S L(2, \mathbb{R})$ which are not bounded below. For instance, one unit spectral flow of the lowest weight representation gives the weight diagram of $\hat{\mathscr{D}}_{j}^{+, w=1}$ shown in Figure 2.

The only case one gets a positive energy representation by the spectral flow is $\hat{\mathscr{D}}_{j}^{ \pm, w=\mp 1}$. Notice that the representations $\widehat{\mathscr{D}}_{\tilde{j}}^{ \pm, w=\mp 1}$ and $\widehat{\mathscr{D}}_{\frac{k}{2}-\tilde{j}}^{\mp, w=0}$ are equivalent. This has an important consequence on the values allowed for $j$. Indeed, $j$ is restricted as required by the no-ghost theorem [15] to

$$
\frac{1}{2}<j<\frac{k-1}{2}
$$

Finally, the complete Hilbert space of string theory on $A d S_{3}$ is obtained by applying creation 
operators $\widetilde{J_{n}^{a}}, n<0$ on the primary states. The physical state conditions are

$$
\begin{aligned}
\left(L_{0}-1\right)|\widetilde{j}, \widetilde{m}, w, \widetilde{N}, h\rangle & =\left(-\frac{\widetilde{j}(\widetilde{j}-1)}{k-2}-w \widetilde{m}-\frac{k}{4} w^{2}+\widetilde{N}+h-1\right)|\widetilde{j}, \widetilde{m}, w, \widetilde{N}, h\rangle=0 \\
L_{n}|\widetilde{j}, \widetilde{m}, w, \widetilde{N}, h\rangle & =\left(\widetilde{L}_{n}-w \widetilde{J}_{n}^{3}\right)|\widetilde{j}, \widetilde{m}, w, \widetilde{N}, h\rangle=0 \quad \text { for } n>0 \quad
\end{aligned}
$$

where $\widetilde{N}$ is the excitation level and $h$ is the conformal weight of the state in the internal theory ${ }^{1}$.

The primary states in the sector $w=0$ can be represented by an operator $\Phi_{j}(x, \bar{x} ; w, \bar{w})$ which satisfies the following OPE with the currents

$$
J^{a}(z) \Phi_{j}(x, \bar{x} ; w, \bar{w}) \sim \frac{D^{a}}{z-w} \Phi_{j}(x, \bar{x} ; w, \bar{w}), \quad(a=3, \pm),
$$

where the differential operators $D^{+}=\frac{\partial}{\partial x}, D^{3}=x \frac{\partial}{\partial x}+j, D^{-}=x^{2} \frac{\partial}{\partial x}+2 j x$, give a representation of the Lie algebra of $S L(2)$. Here $x, \bar{x}$ keep track of the $S L(2)$ weights of the fields and they are interpreted as the coordinates of the boundary in the AdS/CFT context.

Alternatively one can write operators in the $m$ basis through the following transformation from the $x$ basis

$$
\Phi_{j ; m, \bar{m}}=\int \frac{d^{2} x}{|x|^{2}} x^{j-m} \bar{x}^{j-\bar{m}} \Phi_{j}(x, \bar{x})
$$

where $m-\bar{m}$ is an integer.

The spectral flowed states in the sector $w=1$ are constructed by the fusion of $\Phi_{j}$ with the spectral flow operator $\Phi_{\frac{k}{2}}$ through the following operation [17]

$$
\begin{aligned}
\Phi_{J, \bar{J}}^{w=1, j}(x, \bar{x} ; z, \bar{z}) \equiv \lim _{\varepsilon, \bar{\varepsilon} \rightarrow 0} \varepsilon^{m} \bar{\varepsilon}^{\bar{m}} \int d^{2} y y^{j-m-1} \bar{y}^{j-\bar{m}-1} \\
\times \quad \Phi_{j}(x+y, \bar{x}+\bar{y} ; z+\varepsilon, \bar{z}+\bar{\varepsilon}) \Phi_{\frac{k}{2}}(x, \bar{x} ; z, \bar{z}),
\end{aligned}
$$

where $J=m+\frac{k}{2}, \bar{J}=\bar{m}+\frac{k}{2}$, denote the left and right spins of the $w=1$ field. In the $x$ basis, the winding number $w$ turns out to be always positive, unlike in the $m$ basis where the sign of $w$ is correlated with the sign of $m$, thus distinguishing by convention incoming from outgoing spectral flowed states in the correlation functions.

Vertex operators for string states in higher winding sectors can be easily obtained in the $m$ basis where they are expressed in terms of $S L(2)$ parafermions and one free boson [15]. However, as the winding number increases, they become more complicated in the $x$ basis.

\section{Correlation functions in $S L(2, \mathbb{R})$ WZW models}

The correlation functions in WZW models obey linear differential equations which follow from the Sugawara construction of the energy-momentum tensor $T(z)$. The Knizhnik-Zamolodchikov (KZ) equation in $S L(2, \mathbb{R})$, obtained from

$$
\left.\left\langle T(z) \Phi_{j_{1}}\left(z_{1}, x_{1}\right) \cdots \Phi_{j_{N}}\left(z_{N}, x_{N}\right)\right\rangle \rightarrow\left((k-2) L_{1}+J_{-1}^{a} J_{0}^{a}\right)\right)\left\langle\Phi_{j_{1}}\left(z_{1}, x_{1}\right) \cdots \Phi_{j_{N}}\left(z_{n}, x_{N}\right)\right\rangle=0
$$

\footnotetext{
${ }^{1}$ We have been considering string theory on $A d S_{3}$, but more generally we could take a background $A d S_{3} \times \mathscr{N}$, with $\mathscr{N}$ a compact internal manifold.
} 
determines the following equation for $A_{N} \equiv\left\langle\Phi_{j_{1}}\left(x_{1}, z_{1}\right) \Phi_{j_{2}}\left(x_{2}, z_{2}\right) \cdots \Phi_{j_{N}}\left(x_{N}, z_{N}\right)\right\rangle$, an $N$ point function of primary $w=0$ fields,

$$
\begin{aligned}
(k-2) \frac{\partial A_{N}}{\partial z_{i}}=\sum_{n=1, n \neq i}^{N} \frac{1}{z_{i}-z_{n}} & {\left[\left(x_{n}-x_{i}\right)^{2} \frac{\partial^{2}}{\partial x_{i} \partial x_{n}}+\right.} \\
+ & \left.2\left(x_{n}-x_{i}\right)\left(j_{n} \frac{\partial}{\partial x_{i}}-j_{i} \frac{\partial}{\partial x_{n}}\right)-2 j_{i} j_{n}\right] A_{N} .
\end{aligned}
$$

In addition, if $A_{N}$ involves a spectral flow operator, say $\Phi_{\frac{k}{2}}\left(x_{2}, z_{2}\right)$, then it must also obey the following null vector equation

$$
0=\sum_{n=1, n \neq 2}^{N} \frac{x_{n}-x_{2}}{z_{2}-z_{n}}\left[\left(x_{n}-x_{2}\right) \frac{\partial}{\partial x_{n}}+2 j_{n}\right] A_{N},
$$

since $\Phi_{\frac{k}{2}}$ has a null descendant, namely $J_{-1}^{-}|j=k / 2 ; m=k / 2\rangle=0$.

Furthermore, the global $S L(2)$ symmetry of the WZW model determines the Ward identities to be satisfied by the correlation functions

$$
\begin{aligned}
0 & =\sum_{i=1}^{N} \frac{\partial A_{N}}{\partial x_{i}} \\
0 & =\sum_{i=1}^{N}\left(x_{i} \frac{\partial}{\partial x_{i}}+j_{i}\right) A_{N}, \\
0 & =\sum_{i=1}^{N}\left(x_{i}^{2} \frac{\partial}{\partial x_{i}}+2 j_{i} x_{i}\right) A_{N} .
\end{aligned}
$$

(and similarly for $x_{i} \rightarrow z_{i}$ replacing also $j_{i} \rightarrow \Delta_{i}$ ).

Solving these equations, the following amplitudes have been computed so far. Correlation functions of two, three and four unflowed string states have been obtained in [17] performing analytic continuation on the results for the Euclidean $S L(2, \mathbb{C}) / S U(2)$ WZW model obtained in [18, 19]. Actually the Hilbert space of this coset model consists of irreducible representations of $S L(2, \mathbb{C})$ parametrized by $j=\frac{1}{2}+i s, s \in \mathbb{R}_{\geq 0}$. The construction of expectation values of these primary fields in [18] is based on a generalization of the bootstrap approach to this non-RCFT. The structure constants are derived from four point functions involving one degenerate field which satisfy differential equations following from the null vector decoupling condition. Assuming crossing symmetry these four point functions lead to a unique solution for the structure constants of generic primary fields. Expressions for amplitudes of unflowed states have been also written in the $m$-basis, performing the integral transform (3.11) from the $x$-basis results [20,21].

Scattering amplitudes of $n$ - unflowed states in string theory on $A d S_{3}$ exhibit several subtleties for $n \geq 3$. On the one hand, correlation functions of discrete states are only well defined if the sum of the isospins $j$ of the external operators satisfies $\sum_{i} j_{i}<k$. Moreover the four point functions do not factorize as expected into a sum of products of three point functions with physical intermediate states unless the quantum numbers of the external states verify $j_{1}+j_{2}<\frac{k+1}{2}$ and $j_{3}+j_{4}<\frac{k+1}{2}$. The interpretation of these constraints presented in [17] indicates that correlation functions violating 
these bounds do not represent well defined computations in the dual CFT description of the theory on the boundary. This explanation is similar to the interpretation of the upper bound on the spin of the physical states (i.e., $j<\frac{k-1}{2}$ ) as the condition that only local operators be considered in the boundary CFT. However in the later case one has a clear understanding of the constraint from the representations of $S L(2, \mathbb{R})$ which define the theory in the bulk. Similarly one would like to better understand this unusual feature of the correlation functions from the worldsheet viewpoint.

As discussed in the previous section, spectral flowed states are not simply related to states in the coset model by analytic continuation. Consequently two alternative procedures to compute correlation functions involving states in $w \neq 0$ sectors have been discussed in [17]. Starting from $x$-basis correlators of states in the $w=0$ sector which include also spectral flow operators, one can either transform the result to the $m$-basis and perform the spectral flow operation, or one can spectral flow directly in the $x$-basis using the definition (3.12) of one unit spectral flowed states. The following correlation functions involving spectral flowed operators have been computed so far. The two point function of states in arbitrary winding sectors was obtained in reference [17] (see also [4] for a derivation in the $m$ basis using the free field approach) and it is the following

$$
\begin{aligned}
&\left\langle\Phi_{J, J}^{w, j}\left(x_{1}, z_{1}\right) \Phi_{J, \bar{J}}^{w, j^{\prime}}\left(x_{2}, z_{2}\right)\right\rangle=x_{12}^{-2 J} \bar{x}_{12}^{-2 \bar{J}} z_{12}^{-2 \Delta_{j}^{w}} \bar{z}_{12}^{-2 \bar{\Delta}_{j}^{w}} \\
& \times\left[\delta\left(j+j^{\prime}-1\right)+\delta\left(j-j^{\prime}\right) \frac{\pi B(j)}{\gamma(2 j)} \frac{\Gamma(j+m)}{\Gamma(1-j+m)} \frac{\Gamma(j-\bar{m})}{\Gamma(1-j-\bar{m})}\right],
\end{aligned}
$$

where $\Delta_{j}^{w}=-\frac{j(j-1)}{k-2}-m w-\frac{k}{4} w^{2}$ and

$$
B(j)=\frac{k-2}{\pi} \frac{v^{1-2 j}}{\gamma\left(\frac{2 j-1}{k-2}\right)}, \quad v=\pi \frac{\Gamma\left(\frac{k-3}{k-2}\right)}{\Gamma\left(\frac{k-1}{k-2}\right)}, \quad \gamma(x) \equiv \frac{\Gamma(x)}{\Gamma(1-x)} .
$$

Recall that in the $x$ basis the operators are labeled with positive $w$, so this two point function conserves winding number as expected. Indeed, physical amplitudes of $n$ string states may violate winding number conservation up to $n-2$ units. This fact is well understood from the representation theory of $S L(2, \mathbb{R})[17]$.

The three point function including one $w=1$ operator is the following [17] ${ }^{2}$

$$
\begin{aligned}
\left\langle\Phi_{J, \bar{J}}^{w=1, j_{1}}\left(x_{1}, z_{1}\right) \Phi_{j_{2}}\left(x_{2}, z_{2}\right) \Phi_{j_{3}}\left(x_{3}, z_{3}\right)\right\rangle= \\
=B\left(j_{1}\right) C\left(\frac{k}{2}-j_{1}, j_{2}, j_{3}\right) \pi \frac{1}{\gamma\left(j_{1}+j_{2}+j_{3}-k / 2\right)} \\
\quad \times \frac{\Gamma\left(j_{1}+J-\frac{k}{2}\right)}{\Gamma\left(1+J-j_{2}-j_{3}\right)} \frac{\Gamma\left(j_{2}+j_{3}-\bar{J}\right)}{\Gamma\left(1-j_{1}-\bar{J}+\frac{k}{2}\right)} \\
\quad \times\left(x_{21}^{j_{3}-j_{2}-J} x_{31}^{j_{2}-j_{3}-J} x_{32}^{J-j_{2}-j_{3}}\right)\left(z_{21}^{\Delta_{3}-\Delta_{2}-\Delta_{1}^{w=1}} z_{31}^{\Delta_{2}-\Delta_{3}-\Delta_{1}^{w=1}} z_{32}^{\Delta_{1}^{w=1}-\Delta_{2}-\Delta_{3}}\right) \\
\quad \times(\text { antiholomorphic part }),
\end{aligned}
$$

\footnotetext{
${ }^{2}$ Actually this expression differs from the one in [17] by an irrelevant factor $(-1)^{J-\bar{J}}$, as it can be verified using the property $J-\bar{J} \in \mathbf{Z}$ together with the identity $\Gamma(x) \Gamma(1-x)=\frac{\pi}{\sin (\pi x)}$.
} 
where $J=m+k / 2, \Delta_{1}^{w=1}=\Delta_{1}-J+\frac{k}{4}$ and $C\left(j_{1}, j_{2}, j_{3}\right)$ is the coefficient corresponding to the amplitude of three $w=0$ fields, namely

$$
C\left(j_{1}, j_{2}, j_{3}\right)=-\frac{G\left(1-j_{1}-j_{2}-j_{3}\right) G\left(j_{3}-j_{1}-j_{2}\right) G\left(j_{2}-j_{3}-j_{1}\right) G\left(j_{1}-j_{2}-j_{3}\right)}{2 \pi^{2} v^{j_{1}+j_{2}+j_{3}-1} \gamma\left(\frac{k-1}{k-2}\right) G(-1) G\left(1-2 j_{1}\right) G\left(1-2 j_{2}\right) G\left(1-2 j_{3}\right)},
$$

where

$$
G(j)=(k-2)^{\frac{j(k-1-j)}{2(k-2)}} \Gamma_{2}(-j \mid 1, k-2) \Gamma_{2}(k-1+j \mid 1, k-2),
$$

and $\Gamma_{2}(x \mid 1, \omega)$ is the Barnes double Gamma function which reads

$$
\log \left(\Gamma_{2}(x \mid 1, \omega)\right)=\lim _{\varepsilon \rightarrow 0} \frac{\partial}{\partial \varepsilon}\left[\sum_{n, m=0}^{\infty}(x+n+m \omega)^{-\varepsilon}-\sum_{n, m=0 ;(n, m) \neq(0,0)}^{\infty}(n+m \omega)^{-\varepsilon}\right] .
$$

The winding non-conserving three point function (4.9) was obtained in [17] by first computing a four point function including one spectral flow operator $\Phi_{\frac{k}{2}}$. Such calculation is performed by explicitly solving the corresponding $\mathrm{KZ}$ and null vector equations. The four point function gives rise to (4.9) after spectral flowing as in the definition (3.12) or alternatively, after transforming to the $m$ basis, extracting the pole residue at $m=-\frac{k}{2}$ and acting with the spectral flow operator on the unflowed field $\Phi_{j_{1}}$.

The three point function involving two $w=1$ fields was computed in [2]. The starting point is the five point function with two spectral flow operators, namely

$$
A_{5} \equiv\left\langle\Phi_{\frac{k}{2}}\left(x_{1}, z_{1}\right) \Phi_{\frac{k}{2}}\left(x_{2}, z_{2}\right) \Phi_{j_{1}}\left(x_{3}, z_{3}\right) \Phi_{j_{2}}\left(x_{4}, z_{4}\right) \Phi_{j_{3}}\left(x_{5}, z_{5}\right)\right\rangle .
$$

The $x_{i}$ dependence of the solution to the null vector equations was found in [22] (see also [17]). The complete solution, including the dependence on the worldsheet coordinates $z_{i}$, which is determined from the Ward identities and the KZ equations, is the following [2]

$$
\begin{aligned}
A_{5}= & B\left(j_{1}\right) B\left(j_{3}\right) C\left(\frac{k}{2}-j_{1}, j_{2}, \frac{k}{2}-j_{3}\right)\left|z_{12}\right|^{k}\left|z_{13}\right|^{-2 j_{1}}\left|z_{14}\right|^{-2 j_{2}}\left|z_{15}\right|^{-2 j_{3}} \\
& \times\left|z_{23}\right|^{-2 j_{1}}\left|z_{24}\right|^{-2 j_{2}}\left|z_{25}\right|^{-2 j_{3}}\left|z_{34}\right|^{2\left(\Delta_{3}-\Delta_{1}-\Delta_{2}\right)}\left|z_{35}\right|^{2\left(\Delta_{2}-\Delta_{1}-\Delta_{3}\right)}\left|z_{45}\right|^{2\left(\Delta_{1}-\Delta_{2}-\Delta_{3}\right)} \\
& \times\left|x_{12}\right|^{2\left(j_{1}+j_{2}+j_{3}-k\right)}\left|\mu_{1}\right|^{2\left(j_{1}-j_{2}-j_{3}\right)}\left|\mu_{2}\right|^{2\left(j_{2}-j_{1}-j_{3}\right)}\left|\mu_{3}\right|^{2\left(j_{3}-j_{1}-j_{2}\right)}
\end{aligned}
$$

with

$$
\begin{aligned}
& \mu_{1}=\frac{x_{14} x_{25}}{z_{14} z_{25}}-\frac{x_{15} x_{24}}{z_{15} z_{24}}, \\
& \mu_{2}=\frac{x_{15} x_{23}}{z_{15} z_{23}}-\frac{x_{13} x_{25}}{z_{13} z_{25}}, \\
& \mu_{3}=\frac{x_{13} x_{24}}{z_{13} z_{24}}-\frac{x_{14} x_{23}}{z_{14} z_{23}}
\end{aligned}
$$

As an intermediate step before computing the three point function, one can spectral flow once to obtain the following four point function

$$
A_{4}^{w=1}=\left\langle\Phi_{J_{1}, J_{1}}^{w=1, j_{1}}\left(x_{1}, z_{1}\right) \Phi_{\frac{k}{2}}\left(x_{2}, z_{2}\right) \Phi_{j_{2}}\left(x_{4}, z_{4}\right) \Phi_{j_{3}}\left(x_{5}, z_{5}\right)\right\rangle
$$




$$
\begin{aligned}
& =2 i \pi(-1)^{m_{1}+\bar{m}_{1}} B\left(j_{1}\right) B\left(j_{3}\right) C\left(\frac{k}{2}-j_{1}, j_{2}, \frac{k}{2}-j_{3}\right) \gamma\left(j_{2}-j_{1}-j_{3}+1\right) \\
& \times \frac{\Gamma\left(j_{1}-J_{1}+\frac{k}{2}\right) \Gamma\left(j_{3}-j_{2}+\bar{J}_{1}-\frac{k}{2}\right)}{\Gamma\left(1-j_{1}+\bar{J}_{1}-\frac{k}{2}\right) \Gamma\left(j_{2}-j_{3}-J_{1}+\frac{k}{2}+1\right)} \\
& \times \quad\left[{ }_{2} F_{1}\left(j_{1}+j_{2}-j_{3}, j_{1}-J_{1}+\frac{k}{2}, j_{2}-j_{3}-J_{1}+\frac{k}{2}+1 ; u\right)\right. \\
& \times{ }_{2} F_{1}\left(j_{1}+j_{2}-j_{3}, j_{1}-\bar{J}_{1}+\frac{k}{2}, j_{2}-j_{3}-\bar{J}_{1}+\frac{k}{2}+1 ; \bar{u}\right) \\
& +\lambda u^{j_{3}+J_{1}-j_{2}-\frac{k}{2}} \bar{u}^{j_{3}+\bar{J}_{1}-j_{2}-\frac{k}{2}}{ }_{2} F_{1}\left(j_{1}+j_{3}-j_{2}, j_{1}+J_{1}-\frac{k}{2}, j_{3}+J_{1}-j_{2}-\frac{k}{2}+1 ; u\right) \\
& \left.\times{ }_{2} F_{1}\left(j_{1}+j_{3}-j_{2}, j_{1}+\bar{J}_{1}-\frac{k}{2}, j_{3}+\bar{J}_{1}-j_{2}-\frac{k}{2}+1 ; \bar{u}\right)\right] \\
& \times x_{12}^{j_{2}+j_{3}-J_{1}-\frac{k}{2}} \bar{x}_{12}^{j_{2}+j_{3}-\overline{J_{1}}-\frac{k}{2}}\left|x_{14}\right|^{-4 j_{2}} x_{15}^{j_{2}-j_{3}-J_{1}+\frac{k}{2}} \bar{x}_{15}^{j_{2}-j_{3}-\bar{J}_{1}+\frac{k}{2}} \\
& \times x_{25}^{J_{1}-\frac{k}{2}-j_{2}-j_{3}} \bar{x}_{25}^{\bar{J}_{1}-\frac{k}{2}-j_{2}-j_{3}} z_{14}^{\Delta_{3}-\Delta_{1}^{w}-\Delta_{2}-\Delta_{k / 2}} \bar{z}_{14}^{\Delta_{3}-\bar{\Delta}_{1}^{w}-\Delta_{2}-\Delta_{k / 2}} z_{15}^{\Delta_{2}-\Delta_{1}^{w}-\Delta_{3}+\Delta_{k / 2}} \\
& \times \bar{z}_{15}^{\Delta_{2}-\bar{\Delta}_{1}^{w}-\Delta_{3}+\Delta_{k / 2}} z_{45}^{\Delta_{k / 2}+\Delta_{1}^{w}-\Delta_{3}-\Delta_{2}} \bar{z}_{45}^{\Delta_{k / 2}+\bar{\Delta}_{1}^{w}-\Delta_{3}-\Delta_{2}}\left|z_{25}\right|^{k} \\
& \times \quad z^{J_{1}} \bar{z}^{\bar{J}_{1}}|1-u|^{2\left(j_{1}-j_{2}-j_{3}\right)}|1-z|^{-2 j_{2}} \text {. }
\end{aligned}
$$

Here $x=\frac{x_{12} x_{45}}{x_{14} x_{25}}, z=\frac{z_{12} z 45}{z_{14 z_{25}}}, u=\frac{1-x}{1-z}$ and

$$
\begin{gathered}
\lambda=\frac{\gamma\left(j_{1}+j_{3}-j_{2}\right) \Gamma\left(j_{2}-j_{3}-J_{1}+\frac{k}{2}+1\right) \Gamma\left(j_{1}+\bar{J}_{1}-\frac{k}{2}\right)}{\gamma\left(j_{1}+j_{2}-j_{3}\right) \Gamma\left(j_{3}+\bar{J}_{1}-j_{2}-\frac{k}{2}+1\right) \Gamma\left(j_{3}+\bar{J}_{1}-j_{2}-\frac{k}{2}\right)} \\
\times \frac{\Gamma\left(\overline{J_{1}}-\frac{k}{2}-j_{1}+1\right) \Gamma\left(j_{2}-j_{3}-J_{1}+\frac{k}{2}\right)}{\Gamma\left(j_{1}-J_{1}+\frac{k}{2}\right) \Gamma\left(-j_{1}-J_{1}+\frac{k}{2}+1\right)} .
\end{gathered}
$$

This is not a physical correlator, since the spectral flow operator is outside the unitarity bound. However, this auxiliary result is useful for the computation of four point functions involving one spectral flowed and three unflowed generic states that we perform below.

Now, the three point function can be obtained either spectral flowing once more from this four point function or fusing two physical fields in the five point function (4.12), say $\Phi_{j_{1}}\left(x_{3}, z_{3}\right)$ and $\Phi_{j_{2}}\left(x_{4}, z_{4}\right)$, with the spectral flow operators through the prescription (3.12). The final result is

$$
\begin{aligned}
& \left\langle\Phi_{J_{1}, J_{1}}^{w=1, j_{1}}\left(x_{1}, z_{1}\right) \Phi_{J_{2}, J_{2}}^{w=1, j_{2}}\left(x_{2}, z_{2}\right) \Phi_{j_{3}}\left(x_{3}, z_{3}\right)\right\rangle=B\left(j_{1}\right) B\left(j_{2}\right) C\left(\frac{k}{2}-j_{1}, \frac{k}{2}-j_{2}, j_{3}\right) \\
& \times \frac{\Gamma\left(j_{3}-J_{1}+J_{2}\right) \Gamma\left(j_{3}+\bar{J}_{1}-\bar{J}_{2}\right) \Gamma\left(2-j_{1}-j_{2}-j_{3}\right)^{2}}{\Gamma\left(1-j_{3}-J_{1}+J_{2}\right) \Gamma\left(1-j_{3}+\bar{J}_{1}-\bar{J}_{2}\right)} W\left(j_{1}, j_{2}, j_{3}, J_{1}, J_{2}, \bar{J}_{1}, \bar{J}_{2}\right) \\
& \times x_{12}^{j_{3}-J_{1}-J_{2}} \bar{x}_{12}^{j_{3}-\bar{J}_{1}-\bar{J}_{2}} x_{13}^{\left(J_{2}-J_{1}-j_{3}\right)} \bar{x}_{13}^{\bar{z}_{2}-\bar{J}_{1}-j_{3}} x_{23}^{J_{1}-J_{2}-j_{3}} \bar{x}_{23}^{\bar{J}_{1}-\bar{J}_{2}-j_{3}} \\
& \times z_{12}^{\Delta_{3}-\Delta_{1}^{w=1}-\Delta_{2}^{w=1}} \frac{\Delta_{3}-\bar{\Delta}_{1}^{w=1}-\bar{\Delta}_{2}^{w=1}}{z_{12}} z_{13}^{\Delta_{2}^{w=1}-\Delta_{1}^{w=1}-\Delta_{3}} \bar{z}_{12}^{w=1}-\bar{\Delta}_{1}^{w=1}-\Delta_{3} \\
& \times z_{23}^{\Delta_{1}^{w=1}-\Delta_{2}^{w=1}-\Delta_{3}} \overline{\bar{\Delta}}_{23}^{w=1}-\bar{\Delta}_{2}^{w=1}-\Delta_{3},
\end{aligned}
$$

where

$$
W\left(j_{i}, J_{i}, \bar{J}_{i}\right)=s\left(j_{2}-j_{1}-j_{3}\right) G\left[\begin{array}{c}
j_{2}+J_{2}-\frac{k}{2}, j_{2}-j_{1}-j_{3}+1,1-j_{3}+J_{2}-J_{1} \\
j_{2}-j_{1}+J_{2}-J_{1}+1,2-j_{1}-j_{3}+J_{2}-\frac{k}{2}
\end{array}\right]
$$




$$
\begin{gathered}
\times\left\{s\left(j_{1}-j_{2}-j_{3}\right) G\left[\begin{array}{c}
j_{1}-j_{2}-j_{3}+1, j_{1}+\bar{J}_{1}-\frac{k}{2}, 1-j_{3}+\bar{J}_{1}-\bar{J}_{2} \\
2-j_{2}-j_{3}+\bar{J}_{1}-\frac{k}{2}, j_{1}-j_{2}+\bar{J}_{1}-\bar{J}_{2}+1
\end{array}\right]\right. \\
\left.-s\left(1-2 j_{2}\right) G\left[\begin{array}{c}
j_{2}-\bar{J}_{2}+\frac{k}{2}, j_{2}-j_{1}-j_{3}+1,1-j_{3}+\bar{J}_{1}-\bar{J}_{2} \\
j_{2}-j_{1}+\bar{J}_{1}-\bar{J}_{2}+1,2-j_{1}-j_{3}-\bar{J}_{2}+\frac{k}{2}
\end{array}\right]\right\} \\
+s\left(j_{1}-j_{2}-j_{3}\right) G\left[\begin{array}{c}
j_{1}-j_{2}-j_{3}+1, j_{1}-J_{1}+\frac{k}{2}, 1-j_{3}-J_{1}+J_{2} \\
2-j_{2}-j_{3}-J_{1}+\frac{k}{2}, 1+j_{1}-j_{2}+J_{2}-J_{1}
\end{array}\right] \\
\times\left\{-s\left(1-2 j_{1}\right) G\left[\begin{array}{c}
j_{1}-j_{2}-j_{3}+1, j_{1}+\bar{J}_{1}-\frac{k}{2}, 1-j_{3}+\bar{J}_{1}-\bar{J}_{2} \\
2-j_{2}-j_{3}+\bar{J}_{1}-\frac{k}{2}, j_{1}-j_{2}+\bar{J}_{1}-\bar{J}_{2}+1
\end{array}\right]\right. \\
\left.+s\left(j_{2}-j_{1}-j_{3}\right) G\left[\begin{array}{c}
j_{2}-\bar{J}_{2}+\frac{k}{2}, j_{2}-j_{1}-j_{3}+1,1-j_{3}+\bar{J}_{1}-\bar{J}_{2} \\
j_{2}-j_{1}+\bar{J}_{1}-\bar{J}_{2}+1,2-j_{1}-j_{3}-\bar{J}_{2}+\frac{k}{2}
\end{array}\right]\right\},
\end{gathered}
$$

with $G\left[\begin{array}{c}a, b, c \\ e, f\end{array}\right] \equiv \frac{\Gamma(a) \Gamma(b) \Gamma(c)}{\Gamma(e) \Gamma(f)} 3 F_{2}(a, b, c ; e, f ; 1)$ and $s(a)=\sin (\pi a)$.

Let us analyze the properties of this result. The function $W\left(j_{i}, J_{i}, \bar{J}_{i}\right)$ is analytic in its arguments for states belonging to the continuous representation or their spectral flow images. Therefore the three point function (4.16) is perfectly well behaved and finite for normalizable operators with $j=\frac{1}{2}+i s$, as expected. If one of the original unflowed states, say $\Phi_{j_{1}}$, belongs to a lowest weight representation, i.e., $m_{1}=j_{1}+n_{1}, \bar{m}_{1}=j_{1}+\bar{n}_{1}$ with $n_{1}, \bar{n}_{1}=0,1,2 \cdots$, then it can be shown that $W\left(j_{i}, J_{i}, \bar{J}_{i}\right)$ greatly simplifies, and taking further $n_{1}, \bar{n}_{1}=0$ the hypergeometric functions become unity. The analysis of $W\left(j_{i}, J_{i}, \bar{J}_{i}\right)$ completely agrees with that of reference [21] (taking into account the change in notation). However notice that we are dealing with a winding conserving three point function which includes two one unit spectral flowed states whereas [21] considers unflowed states. Moreover (4.16) is an $x$ basis correlator unlike the $m$ basis expression analyzed in [21].

The three point function (4.16) has various poles which come from the poles in $C_{5}$, in the $\Gamma$-functions and in the unrenormalized hypergeometric functions. $C_{5}$ has the same poles as the unflowed three point function, namely at

$$
j=n+m(k-2), \quad-(n+1)-(m+1)(k-2), \quad n, m=0,1,2, \cdots \quad,
$$

with

$$
j=1-j_{1}-j_{2}-j_{3}, \quad j_{1}-j_{2}-j_{3}, \quad j_{2}-j_{3}-j_{1}, \quad j_{3}-j_{2}-j_{1} .
$$

The $\Gamma$-functions add the following poles

$$
J_{1}=J_{2}+j_{3}+n, \quad J_{2}=J_{1}+j_{3}+n,
$$

and similar ones for $\bar{J}_{1}, \bar{J}_{2}$. The poles of $G\left[\begin{array}{c}a, b, c \\ e, f\end{array}\right]$ are at $a, b, c, u=-n$, with $u=e+f-a-b-c$, and thus they are all contained in the previous ones except for the poles signaling the presence of spectral flowed images of the discrete representations, e.g. $m_{1}=j_{1}+n_{1}, \bar{m}_{1}=j_{1}+\bar{n}_{1}$. Therefore the pole structure is as discussed in reference [17] in the unflowed case with the addition of (4.20), which are analogous to poles in the $S$ matrix of string theory in Minkowski space. 
The computation of more complicated correlation functions along the lines discussed above would require to start from higher point amplitudes. Actually the cases following in complexity, namely the three point function including three one-unit spectral flowed operators or the four point function involving one $w=1$ field require the knowledge of the six point function with three spectral flow operators and three physical states or the five point function with one $\Phi_{\frac{k}{2}}$ and four generic unflowed fields respectively. Therefore, it seems important to find an alternative method to compute such more complicated amplitudes. In the following sections we explore two possible paths, namely general properties of correlation functions containing $w=1$ spectral flowed operators in the $x$ basis and we discuss the free field approximation, a convenient way to compute correlation functions which, so far, seems to reproduce the known exact results.

\subsection{Ward identities, modified $\mathrm{KZ}$ and null vector equations}

The form of the Ward identities for correlation functions involving one unit spectral flowed fields was investigated in [2]. Using the definition (3.12) for the $w=1$ field, it was shown that they maintain the standard form (4.6) with the obvious replacements for the spin and conformal weight of the field $\Phi_{J, \bar{J}}^{w=1, j}$, i.e. $j_{i} \rightarrow J_{i}=m_{i}+\frac{k}{2}$ and $\Delta_{i} \rightarrow \Delta_{i}-J_{i}+\frac{k}{4}$. The analysis can be generalized to correlation functions including an arbitrary number of $w=1$ states. From here, the general form of the two and three point functions containing $w=1$ fields is completely determined, whereas the four point functions depend, as usual, on the anharmonic ratios.

The $\mathrm{KZ}$ and null vector equations for correlators including $w=1$ fields, instead, suffer an important modification. They become iterative expressions in the spin and conformal dimension of the spectral flowed states. Indeed, the modified KZ equation turns out to be

$$
\begin{aligned}
(k-2) \frac{\partial A_{N}^{w}(J)}{\partial z_{i}}= & -\left(j_{1}-J+\frac{k}{2}-1\right) \frac{x_{2}-x_{i}}{\left(z_{i}-z_{2}\right)^{2}}\left[\left(x_{2}-x_{i}\right) \frac{\partial}{\partial x_{i}}-2 j_{i}\right] A_{N}^{w}(J+1) \\
+ & \frac{1}{z_{i}-z_{2}}\left[\left(x_{2}-x_{i}\right)^{2} \frac{\partial^{2}}{\partial x_{i} \partial x_{2}}+\right. \\
& \left.\quad+2\left(x_{2}-x_{i}\right)\left(J \frac{\partial}{\partial x_{i}}-j_{i} \frac{\partial}{\partial x_{2}}\right)-2 j_{i} J\right] A_{N}^{w}(J) \\
+ & \sum_{n=3, n \neq i}^{N+1} \frac{1}{z_{i}-z_{n}}\left[\left(x_{n}-x_{i}\right)^{2} \frac{\partial^{2}}{\partial x_{i} \partial x_{n}}+\right. \\
& \left.\quad+2\left(x_{n}-x_{i}\right)\left(j_{n} \frac{\partial}{\partial x_{i}}-j_{i} \frac{\partial}{\partial x_{n}}\right)-2 j_{i} j_{n}\right] A_{N}^{w}(J)
\end{aligned}
$$

for $A_{N}^{w} \equiv\left\langle\Phi_{m+\frac{k}{2}, \bar{m}+\frac{k}{2}}^{w=1, j_{1}}\left(x_{2}, z_{2}\right) \Phi_{j_{3}}\left(x_{3}, z_{3}\right) \cdots \Phi_{j_{N}}\left(x_{N}, z_{N}\right)\right\rangle$. The notation $A_{N}^{w}(J+1)$ indicates that one must replace $J \stackrel{J}{\longrightarrow}+1$ in $A_{N}^{w}$. Thus, Eq.(4.21) differs from the standard KZ equation for correlators of unflowed fields. In fact, it is an iterative relation in the spin of the spectral flowed field. This feature is not surprising, since it is inherited from the following primary state condition for states in the $w=1$ sector

$$
J\left(x^{\prime}, z^{\prime}\right) \Phi_{J, \bar{J}}^{w=1, j}(x, z)=-(j-m-1) \frac{\left(x-x^{\prime}\right)^{2}}{\left(z^{\prime}-z\right)^{2}} \Phi_{J+1, \bar{J}}^{w=1, j}(x, z)
$$




$$
+\frac{1}{z^{\prime}-z}\left[\left(x-x^{\prime}\right)^{2} \frac{\partial}{\partial x}+2\left(m+\frac{k}{2}\right)\left(x-x^{\prime}\right)\right] \Phi_{J, \bar{J}}^{w=1, j}(x, z),
$$

where $J(x, z)=-J^{-}(z)+2 x J^{3}(z)-x^{2} J^{+}(z)$.

Following a similar procedure with the null vector equation (4.3), one obtains an additional iterative equation, namely

$$
\left(j_{1}+J-\frac{k}{2}-1\right) A_{N}^{w}(J-1)=\sum_{n=3}^{N+1} \frac{x_{n}-x_{2}}{z_{2}-z_{n}}\left[\left(x_{n}-x_{2}\right) \frac{\partial}{\partial x_{n}}+2 j_{n}\right] A_{N}^{w}(J),
$$

which is understood as the modified null vector equation to be satisfied by correlators containing one $w=1$ field. It supplements (4.21), so that both equations must be solved in order to find the explicit expression for $A_{N}^{w}$. These results extend to correlators including any number of $w=1$ fields, where the spins of all the spectral flowed fields turn out to be iterative variables.

\subsection{Four point function including one $w=1$ field}

The modified KZ and null vector equations were explicitly solved in [2] for the four point function involving one $w=1$ field, namely

$$
A_{4}^{w}=\left\langle\Phi_{j_{1}}\left(x_{1}, z_{1}\right) \Phi_{j_{2}}\left(x_{2}, z_{2}\right) \Phi_{J, \bar{J}}^{w=1, j_{3}}\left(x_{3}, z_{3}\right) \Phi_{j_{4}}\left(x_{4}, z_{4}\right)\right\rangle .
$$

From the arguments above, one expects that $A_{4}^{w}$ had the same functional form as an unflowed four point function, but with the spin and conformal dimension of the $w=1$ field given by $J=$ $m+\frac{k}{2}, \Delta_{3}^{w=1}=\Delta_{3}-J+\frac{k}{4}$, respectively. Thus, we consider the following expression for $A_{4}^{w}$

$$
\begin{aligned}
A_{4}^{w} & =\int d j B\left(j_{3}\right) C\left(j_{1}, j_{2}, j\right) B(j)^{-1} C\left(j, \frac{k}{2}-j_{3}, j_{4}\right) \\
& \times D_{1}\left(j_{1}, j_{2}, j_{3}, J, j_{4}, j\right) D_{2}\left(j_{1}, j_{2}, j_{3}, \bar{J}, j_{4}, j\right) \mathscr{F}(z, x) \overline{\mathscr{F}}(\bar{z}, \bar{x}) \\
& \times\left(x_{43}^{j_{1}+j_{2}-j_{4}-J} x_{42}^{-2 j_{2}} x_{41}^{J+j_{2}-j_{4}-j_{1}} x_{31}^{j_{4}-j_{1}-j_{2}-J}\right) \\
& \times\left(z_{43}^{\Delta_{1}+\Delta_{2}-\Delta_{4}-\Delta_{3}^{w=1}} z_{42}^{-2 \Delta_{2}} z_{41}^{\Delta_{3}^{w=1}+\Delta_{2}-\Delta_{4}-\Delta_{1}} z_{31}^{\Delta_{4}-\Delta_{1}-\Delta_{2}-\Delta_{3}^{w=1}}\right) \\
& \times \text { (antiholomorphic part) },
\end{aligned}
$$

where the dependence in the coefficients $B$ and $C$ is inherited from the five point function involving one spectral flow operator, $D_{1}$ and $D_{2}$ are the parts of the coefficient of the four point function depending respectively on the right and left spins of the string states, whereas $\mathscr{F}$ and $\overline{\mathscr{F}}$ are functions of the cross ratios $z=\frac{z_{21} z_{43}}{z_{31} z_{42}}, x=\frac{x_{21} x_{43}}{x_{31} x_{42}}$.

Now plugging (4.25) into the modified $\mathrm{KZ}$ and null vector equations, one finds iterative expressions which can be solved following a similar route to that in the unflowed case $[17,19]$. Namely, expand $\mathscr{F}$ in powers of $z$ as follows

$$
\mathscr{F}(z, x)=z^{\Delta_{j}-\Delta_{j_{1}}-\Delta_{j_{2}}} x^{j-j_{1}-j_{2}} \sum_{n=0}^{\infty} f_{n}(x) z^{n},
$$


and focus on the lowest order of this expansion. Considering first the $\mathrm{KZ}$ equation (4.21), the following solution is found ${ }^{3}$

$$
f_{0}={ }_{2} F_{1}\left(j-j_{1}+j_{2}, j+J-j_{4}, 2 j ; x\right),
$$

where ${ }_{2} F_{1}$ is the standard hypergeometric function. The modified null vector equation (4.23) in turn, allows to find iterative relations for $f_{n}$ in terms of $f_{n-1}$ (for $n \geq 1$ ). However, the coefficients $D_{1}, D_{2}$ cannot be determined in this way since (4.23) gives the following iterative relation

$$
\left(j_{3}+J-\frac{k}{2}-1\right) D_{1}(J-1)=\left(J-j-j_{4}\right) D_{1}(J)
$$

and an analogous expression for $D_{2}$. This means that the modified $\mathrm{KZ}$ and null vector equations do not completely specify the spin dependence of the four point function. This is not surprising since a similar situation is found in the unflowed case. Nevertheless, we are still able to find a proper expression for the coefficient by requiring the following two conditions: $i$ ) that it satisfies (4.28) (and a similar expression for $D_{2}$ ), and ii) that $A_{4}^{w}$ in (4.25) correctly reduces to (4.9), the three point function involving one spectral flowed field, when one of the unflowed operators is the identity.

It can be shown that a solution to $i$ ) and $i$ ) is given by

$$
D_{1} D_{2} \sim \frac{1}{\gamma\left(j_{1}+j_{2}+j_{3}+j_{4}-\frac{k}{2}\right)} \frac{\Gamma\left(j_{3}+J-\frac{k}{2}\right)}{\Gamma\left(1+J-j_{4}-j\right)} \frac{\Gamma\left(j_{4}+j-\bar{J}\right)}{\Gamma\left(1-j_{3}-\bar{J}+\frac{k}{2}\right)},
$$

up to a $k$ dependent coefficient. However this solution is not unique. Such residual uncertainties might be removed studying the factorization properties of the four point function (4.25), following a similar path to that of section 4 in reference [17] for the unflowed case. However here the pole structure of the four point function presents additional difficulties since there are poles in the integral in the complex $j$ plane crossing the integration contour even before performing the analytic continuation and thus this analysis has not been completed.

This summarizes the already known explicit expressions for correlators including spectral flowed fields. Note that, whereas the two point function is known for fields in unlimited winding sectors, the situation gets more complicated in the case of the three point function, where only the case involving one $w=1$ and two $w=0$ operators and that including two $w=1$ and one $w=0$ fields have been computed so far. Moreover, the four point function is only known for unflowed states. The increasing difficulties to compute correlation functions including additional spectral flowed fields are due to the fact that one has to start from amplitudes containing more spectral flow operators.

Therefore it seems necessary to develop techniques that simplify these computations and allow to perform others that would clarify the full structure of the model. The free field description of the theory appears as a powerful tool in this direction, thus in the following section we discuss the status of this approximation.

\footnotetext{
${ }^{3}$ Actually the solution is a linear combination of the functions ${ }_{2} F_{1}\left(j-j_{1}+j_{2}, j+J-j_{4}, 2 j ; x\right)$ and $x^{1-2 j}{ }_{2} F_{1}(1-$ $\left.j-j_{1}+j_{2}, 1-j+J-j_{4}, 2-2 j ; x\right)$. However, analogously as in the unflowed case [17], we may use the fact that, when inserted in (4.25), the two solutions are related to each other through the symmetry $j \longrightarrow 1-j$ which allows to keep only the first solution provided that in (4.11) we now integrate $j$ over the entire imaginary axis, i.e. $\frac{1}{2}+i \mathbf{R}$.
} 


\section{Free field approximation}

The free field approximation to string theory on $A d S_{3}$ was initially applied in [13,14] to derive the spacetime CFT and establish the conjectured AdS/CFT correspondence in the three dimensional case. Even though this approach is expected to give a good picture of the theory only near the boundary of $A d S_{3}$, the computation of two and three point amplitudes of string states using the Coulomb gas formalism in $[3,4]$ has produced results in complete agreement with the exact ones. Moreover, the analysis of unitarity in this approximation might give important information on the consistency of the complete theory. For that reason, the aim of this section is to discuss the current status of this approach.

The assumption that conformal field theories in general should admit a representation in terms of free field operators, with some reduction in the Fock spaces of states, governed by the BRST structure [23], is very attactive. However the extension of the free field representation to nonrational models presents several complications. Actually, as stressed in [23] there are different levels in the free field realization of a CFT. In the case of $S L(2, \mathbb{R})$, the well known Wakimoto construction [24] successfully gives the current algebra. The representation for the primary and screening operators was originally given in [25]. The next step would be to construct the conformal blocks, as specified Fock-space expectation values, which results then in an integral representation of particular analytic functions. Finally, the correlation functions are to be constructed out of the conformal blocks, and the operator algebra of primary operators is to be derived. The theory is then fully solved. Minimal models and $S U(2)$ WZW CFT are prototypical examples where this program has been completed. Actually, in the compact $S U(2)$ case, the characters and fusion rules were successfully reproduced in the free field approach developed by Griffin and Hernandez [26]. Expectation values of $S U(2)$ primary fields on the plane were computed in an alternative free field realization in terms of minimal models in [8]. While this alternative strategy was successfully applied to $S L(2, \mathbb{R})$ by relating it to Liouville correlators [27], the standard Feigin-Fuchs formalism failed to reproduce the spectrum and fusion rules of unitary representations in $S L(2, \mathbb{R})[28]^{4}$. Moreover, contrary to extended expectations [31], the Hilbert space of physical states of string theory on $\mathrm{AdS}_{3}$ constructed as the BRST cohomology on the Fock space of free fields in [32], presents several differences with the spectrum proposed by Maldacena and Ooguri in [15]. This is not surprising since there are no singular vectors in the relevant representations associated to the physical states of string theory on $\mathrm{AdS}_{3}$ [33].

Nevertheless, the explicit computation of the partition function [34] seems to support the idea that the spectrum can be cast in terms of free fields. Furthermore, free field methods also seem to reproduce the exact results for correlation functions. Actually, both the functional integration used in reference [35] and the Coulomb gas formalism implemented in reference [36] for two and three point functions of unflowed states, give results in complete agreement with the expresions found in [18, 19] (see [21]). Moreover, the Coulomb gas construction developed in [3] to deal with correlation functions of spectral flowed states, was applied in [4] to compute two and three point functions, both conserving and violating winding number, and the results also agree with the exact

\footnotetext{
${ }^{4}$ Correlation functions in the $S L(2, \mathbb{R})$ WZW model have been computed in terms of free fields in references [29, 30] for the hamiltonian reduction and fractional levels of the algebra.
} 
computations in [17]. So the questions about how far and why these coincidences hold seem worth being investigated further.

\section{Conclusions}

There are several reasons why it is important to understand string propagation in non-compact backgrounds. In particular, strings moving in $A d S_{3}$, one of the simplest examples beyond flat spacetime, are not well understood yet. Several consistency checks have been performed to determine consistency and unitarity of this theory, but the spectral flow sector needs to be studied further in order to definitely settle these questions.

Furthermore, it seems to be of great significance to develop new techniques to compute correlation functions in this theory, since this would not only ultimately establish the consistency of the model, but it could also enlighten more general questions regarding non-rational CFT. Actually, unlike RCFT, the naive free field representation of $\operatorname{SL}(2, \mathbb{R})$ does not seem to reproduce the spectrum obtained using algebraic methods. However, it seems possible to interprete the partition function in terms of free field contributions and moreover, correlation functions of two and three string states computed in the Coulomb gas formalism seem to agree with the exact results. The resolution of this puzzle might give important clues on these matters. 


\section{References}

[1] V. Schomerus, Non-compact sring backgrounds and non-rational CFT, Phys. Rept. 431 (2006) 39-86; hep-th/0509155.

[2] E. Herscovich, P. Minces and C. Núñez, Winding strings in $A d S_{3}$, JHEP0606 (2006) 047; hep-th/0512196.

[3] G. Giribet and C. Núñez, Aspects of the free field representation of string theory on AdS 3 , JHEP 0005 (2000); hep-th/0006070.

[4] G. Giribet and C. Nuñez, "Correlators in $A d S_{3}$ string theory" JHEP 0106 (2001),hep-th/0105200.

[5] D. Hofman and C. Núñez, Free field realization of superstring theory on $A d S_{3}$, JHEP 007 (2004) 019; hep-th/0404214.

[6] L. Nicolás and C. Núñez, Screening the light in $A d S_{3}$, work in progress.

[7] A. Belavin, A. Polyakov and A. Zamolodchikov, Infinite conformal symmetry in two-dimensional quantum field theory Nucl. Phys. B241 (1984) 333-380.

[8] V. A. Fateev and A. B. Zamoldchikov, Operator algebra and correlation functions in the two dimensional Wess-Zumino Su(2) ×Su(2) chiral model, Sov. J. Nucl. Phys. 43 (1986) 657.

[9] B. L. Feigin and D. B. Fuchs, Func. Anal. Appl.16 (1982) 114; Func. Anal. Appl. 17 (1983) 241.

[10] V.S. Dotsenko y V.A. Fateev,Four-point Correlation Functiones and the Operator Algebra in 2D Conformal Invariant Theories with Central Charge $C \leq 1$ Nucl. Phys. B 251 (1985) 691; V.S. Dotsenko y V.A. Fateev,Conformal Algebra and Multipoint Correlation Functions in Two Dimensional Statistical Models, Nucl. Phys. B 240 (1984) 312.

[11] G. Felder, BRST approach to minimal methods, Nucl. Phys. B317 (1989) 215.

[12] D. Bernard and G. Felder, Fock representations and Brst cohomology in Sl(2) current algebra, Commun. Math. Phys. 127 (1990) 145.

[13] A. Giveon, D. Kutasov and N. Seiberg, Comments on string theory on $A d S_{3}$, Adv. Theor. Math. Phys. 2 (1998) 733, hep-th/9806194.

[14] D. Kutasov and N. Seiberg, More comments on string theory on $A d S_{3}$, JHEP 9904 (1999) 008; hep-th/9903219.

[15] J. Maldacena and H. Ooguri,Strings in $A d S_{3}$ and the $S L(2, \mathbb{R})$ WZW Model. Part 1: The Spectrum, J. Math. Phys. 42 (2001) 2929, hep-th/0001053.

[16] J. Maldacena, H. Ooguri and J. Son,Strings in $A d S_{3}$ and the $S L(2, \mathbb{R})$ WZW Model. Part 2: Euclidean black hole, J. Math. Phys. 42 (2001) 2961, hep-th/0005183.

[17] J. Maldacena and H. Ooguri,Strings in $A d S_{3}$ and the $S L(2, \mathbb{R})$ WZW Model. Part 3: Correlation Functions, Phys.Rev. D65 (2002) hep-th/011180.

[18] J. Teschner, On Structure constants and fusion rules in $S L(2, C) / S U(2) W Z N W$ model, Nucl. Phys. B 546 (1999) 390; hep-th/9906215.

[19] J. Teschner, Operator product expansion and factorization in the $\mathrm{H}_{3}^{+}$WZNW model, Nucl. Phys. B 571 (2000) 555; hep-th/9906215.

[20] A. Giveon and D. Kutasov, Little string theory in a double scaling limit, JHEP 9910 (1999) 034; hep-th/9909110; Comments on double scaled little string theory, JHEP 0001 (2000) 023; hep-th/9911039. 
[21] Y. Satoh, Three point functions and operator product expansion in the SL(2) conformal field theory, Nucl. Phys. B629 (2002) 188.

[22] Fateev and Zamolodchikov, Unpublished notes.

[23] V. S. Dotsenko, The free field representation of the su(2) conformal field theory, Nucl. Phys. $\mathbf{B 3 3 8}$ (1990) 747.

[24] M. Wakimoto, Fock representations of the affine Lie algebra $A_{1}^{(1)}$, Commun. Math. Phys. 104 (1986) 605.

[25] A. Gerasimov, A. Marshakov, A. Morozov, M. Olshanetsky and S. Shatashvili, Wess-Zumino-Witten model as a theory of free fields, Int. J. Mod. Phys. A 5(1990) 2495-2590.

[26] P. Griffin and O. Hernandez, Structure of irreducible SU(2) parafermion modules derived via the Feigin-Fuchs construction, Int. J. Mod. Phys. A7 (1992) 1233-1266.

[27] O. Andreev, Operator algebra of the SL(2) conformal field theories, Phys. Lett. B363 (1995) 166-172

[28] P. Griffin and O. Hernandez, Feigin-Fuchs derivation of SU(1,1) parafermion characters, Nucl. Phys. B356 (1991) 287-300.

[29] P. Furlan, A. Ganchev and V. Petkova, A(1|1) admissible representations: Fusion transformations and local correlators, Nucl. Phys. B491 (1997) 635-658.

[30] J. Petersen, J. Rassmussen and M. Yu, Hamiltonian reduction of SL(2) theories ar the level of correlators, Nucl. Phys. B457 (1995) 343-356.

[31] A. Giveon and D. Kutasov, Notes on $A d S_{3}$, Nucl. Phys. B 621 (2002) 303; hep-th/0106004.

[32] Y. Hikida, K. Hosomichi and Y. Sugawara, "String theory on $A d S_{3}$ as discrete light-cone Liouville theory", (2000) hep-th/00005065.

[33] B. Feigin, D. Fuchs and F. Malikov, Singular vectors in Verma modules over affine Kac-Moody algebras, Func. An. App. 20 (1986) 103-113.

[34] D. Israel, C. Kounnas and P. Petropoulos, Superstrings on NS5 backgrounds, deformed AdS(3) and holography, JHEP 0310 (2003) 028; hep-th/0306053.

[35] N. Ishibashi, K. Okuyama and Y. Satoh, Path integral approach to string theory on AdS 3 , Nucl. Phys. B 588 (2000) 149-177; hep-th/0005152; K. Hosomichi, K. Okuyama and Y. Satoh, Free field approach to string theory on $A d S_{3}$, Nucl. Phys. B 598 (2001) 451; hep-th/0009107.

[36] M. Becker and K. Becker, "Interactions in the $S L(2, \mathbb{R}) / U(1)$ Black Hole Background", Nucl. Phys. B 418 (1994) 206-230, hep-th/9310046.

[37] S. Ribault, Knizhnik-Zamolodchikov equations and spectral flow in AdS 3 string theory, JHEP 0509 (2005) 045. 